\title{
Primordial Nucleosynthesis in the Precision era for Cosmology
}

\author{
Gianpiero Mangano*† \\ Dipartimento di Scienze Fisiche, Universita' di Napoli Federico and INFN, Sezione di \\ Napoli, Italy \\ E-mail: manganoona.infn. ițít
}

ABSTRACT: We review present information on cosmological neutrinos, and more generally on relativistic degrees of freedom at the Cosmic Microwave Background formation epoch, in view of the recent results of WMAP collaborations on temperature anisotropies of the $\mathrm{CMB}$, as well as of recent detailed analysis of Primordial Nucleosynthesis.

\section{Introduction}

We are pretty confident that our Universe is presently filled with quite a large number of neutrinos, of the order of $100 \mathrm{~cm}^{-3}$ for each flavor, despite of the fact that there are no direct evidences for this claim and, more sadly, it will be also very hard to achieve this goal in the future. However several stages of the evolution of the Universe have been influenced by neutrinos, and their silent contribution has been first communicated to other species via weak interactions, and eventually through their coupling with gravity. In fact, Big Bang Nucleosynthesis (BBN), the Cosmic Microwave Background (CMB) and the spectrum of Large Scale Structure (LSS) keep traces of their presence, so that by observing the power spectrum $P(k)$, the photon temperature-temperature angular correlation, and primordial abundances of light nuclei, we can obtain important pieces of information on several features of the neutrino background, as well as on some fundamental parameters, such as their mass scale. It is astonishing, at least for all those of the elementary particle community who moved to "astroparticle" physics, to see that in fact the present bound on the neutrino mass, order $1 \mathrm{eV}$, obtained by studying their effect on suppressing structure formation at small scales, is already stronger than the limit obtained in terrestrial measurement from ${ }^{3} \mathrm{H}$ decay.

${ }^{*}$ Speaker.

${ }^{\dagger}$ Work done in collaboration with A. Cuoco, F. Iocco, G. Miele, O. Pisanti, and P. Serpico. 
In this short lecture I briefly review some of the cosmological observables which indeed lead to relevant information on both dynamical (number density, chemical potential) and kinematical (masses) neutrino properties, as well as on extra weakly coupled light species.

\section{Cosmological neutrinos: standard features}

For large temperatures neutrinos are kept in thermodynamical equilibrium with other species, namely $e^{-}-e^{+}$and nucleons, which in turn share the very same temperature of photons because of electromagnetic interactions. The key phenomenon for cosmological neutrinos is that for temperatures of the order of $T_{d} \sim 1 \mathrm{MeV}$ weak interactions become unable to sustain equilibrium, since the corresponding effective rate $\Gamma_{w}$ (cross-section $\sigma_{w}$ times electron number density $n_{e}$ ) falls below the expansion rate $H$, the Hubble parameter. We can in fact estimate $\sigma_{w} \sim G_{F}^{2} T^{2}$ and $n_{e} \sim T^{3}$, so that $\Gamma_{w}=G_{F}^{2} T^{5}$, and since for a radiation dominated universe $H \sim \sqrt{G_{N}} T^{2}$, with $G_{N}$ the Newton constant ${ }^{1}$ it is straightforward to get

$$
T_{d} \sim \frac{G_{N}^{1 / 6}}{G_{F}^{2 / 3}} \sim 1 M e V
$$

From this epoch on, neutrinos freely stream with an (almost) perfect Fermi-Dirac distribution, the one they had at decoupling, while momentum red-shifts as expansion goes on. In terms of the comoving momentum $y=k a$, with $a$ the scale factor,

$$
d n_{\nu}=a^{-3} \frac{1}{e^{y}+1} \frac{d^{3} y}{(2 \pi)^{3}}
$$

Actually neutrinos are slightly heated up during the $e^{-}-e^{+}$annihilation phase, which takes place at temperatures of the order of the electron mass and release entropy mainly to photons, but also to neutrinos. This is because the neutrino decoupling is not an instantaneous phenomenon, but it partially overlaps the $e^{-}-e^{+}$annihilation phase. A detailed analysis, which also takes into account QED plasma effects on the $e^{-}-e^{+}$pairs [i] shows that neutrino distribution is slightly different than a pure black body distribution, and the corresponding energy density differ from the instantaneous decoupling result at the level of percent.

It is customary to parameterize the contribution $\rho_{R}$ of relativistic species to the expansion rate of the universe in terms of the effective neutrino number $N_{\text {eff }}$ defined as follows

$$
\rho_{R}=\rho_{\gamma}+\rho_{\nu}+\rho_{X}=\left[1+\frac{7}{8}\left(\frac{4}{11}\right)^{4 / 3} N_{\text {eff }}\right] \rho_{\gamma}
$$

with $\rho_{\gamma}, \rho_{\nu}$ and $\rho_{X}$ the energy density of photons, neutrinos and of extra (unknown) light species, respectively. The two factors $7 / 8$ and $(4 / 11)^{4 / 3}$ are due to the difference in the form of equilibrium distribution (Bose-Einstein for photons, Fermi-Dirac for neutrinos) and to the different temperature of photons and neutrinos after $e^{+}-e^{-}$pair annihilation. With this definition, three massless neutrinos with a pure equilibrium distribution and zero

\footnotetext{
${ }^{1}$ I adopt the standard unit system $\hbar=c=k=1$
} 
chemical potential give $N_{e f f}=3$. In view of the partial neutrino reheating from $e^{+}-e^{-}$ the actual value is slightly larger $N_{e f f}=3.04$. The role of this parameter is crucial in our understanding of fundamental physics. Any result in favor of a larger (or a smaller) value for $N_{\text {eff }}$ would imply some exotic non standard physics at work in the early universe. In the following Sections we will see how this parameter is in fact constrained by some crucial cosmological observables, such as $\mathrm{CMB}$ or BBN.

\section{3. $\Omega_{b} h^{2}$ and $N_{e f f}$ after WMAP}

The peak structure of the CMB power spectrum has been beautifully confirmed by a series of experiment in the past few years (BOOMERanG, MAXIMA, DASI, CBI, ACBAR) and more recently by WMAP collaboration [2]2] with a very high accuracy. The improvement in angular resolution from the 7 degrees across the sky of COBE to the order 0.5 degrees of WMAP, allows us to have a better understanding of several features of our Universe and in particular of its matter-energy content in terms of cosmological constant, dark matter and baryons.

The role of relativistic species at the CMB formation, at redshifts of the order of $z \sim 1100$, is mainly to shift the matter radiation equality time, which results in both shifting the peak location in angular scale and changing the power around the first acoustic peak. This is basically due to a change in the early integrated Sachs-Wolfe effect. Several groups have studied this topics, obtaining comparable bounds on $N_{\text {eff }}$ but using different

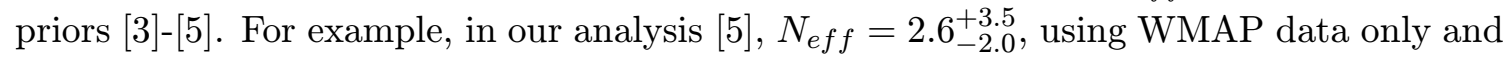
weak prior on the value of the Hubble parameter, $h=0.7 \pm 0.2$. The reason for such a wide range for $N_{e f f}$ is ultimately due to the many unknown cosmological parameters which determine the power spectrum, and in particular to the presence of several degeneracies, i.e. the fact that different choices for some of these parameters produce the very same power spectrum. As an example if we increase both $N_{e f f}$ and the amount of dark matter $\Omega_{c d m}$ we can obtain the same power spectrum provided we do not change the radiation-matter equality. This is shown in Fig.1, a plot of the bi-dimensional likelihood contours in the $N_{e f f}-\Omega_{c d m}$ plane

The baryon density parameter $\Omega_{b} h^{2}$ can be much more severely constrained from the power spectrum. Increasing the baryons in the plasma enhances the effective mass of the fluid and this leads to more pronounced compression peaks. By a likelihood analysis the bound obtained in [i $\left[\begin{array}{l}0 \\ 0\end{array}\right]$ is $\Omega_{b} h^{2}=0.023 \pm 0.002$ ( $1-\sigma$ error), fully compatible with the one quoted by the WMAP Collaboration, $\Omega_{b} h^{2}=0.022 \pm 0.001$, [i2]. In Fig.2 we show the likelihood contours in the $N_{e f f}-\Omega_{b} h^{2}$ plane. This result is extremely important. The WMAP data tell us the value of baryon density with a better accuracy than BBN, so we can test the standard scenario of light nuclei formation with basically no free parameters but the value of $N_{\text {eff }}$.

\section{4. $\Omega_{b} h^{2}$ and $N_{e f f}$ and Big Bang Nuclesynthesis}

The primordial production of light nuclei, mainly ${ }^{4} \mathrm{He}, \mathrm{D}$ and ${ }^{7} \mathrm{Li}$, takes place when the 


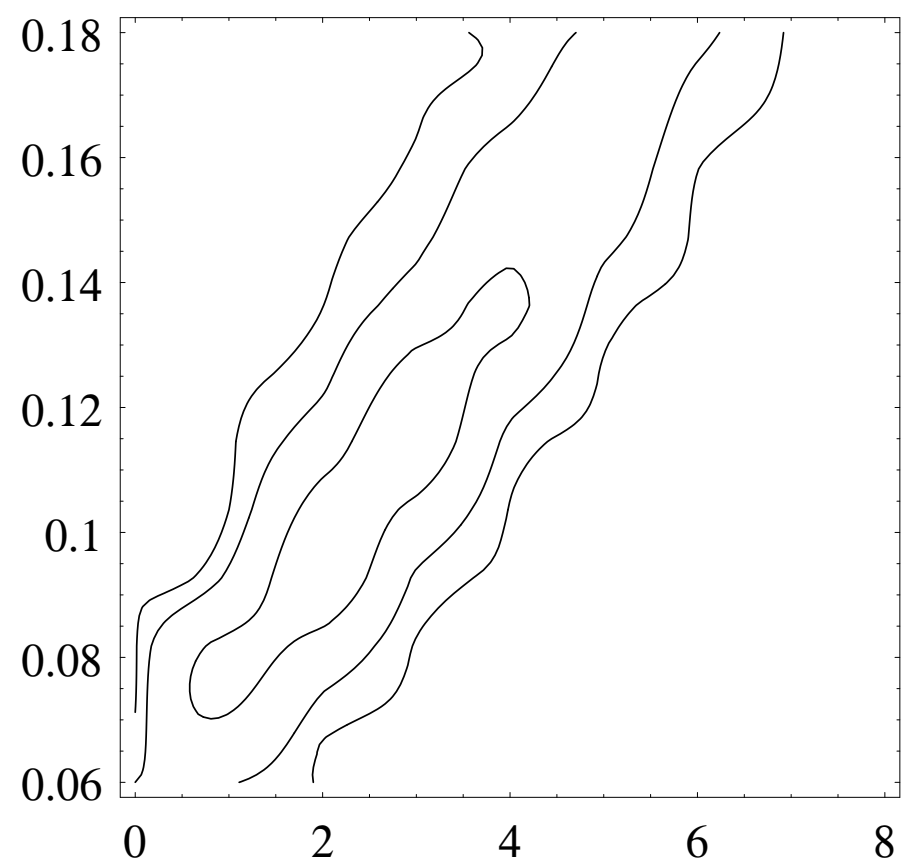

Figure 1: Likelihood contours from WMAP data in the $N_{\text {eff }}$ (x-axis) $-\Omega_{c d m} h^{2}$ (y-axis) plane 演.

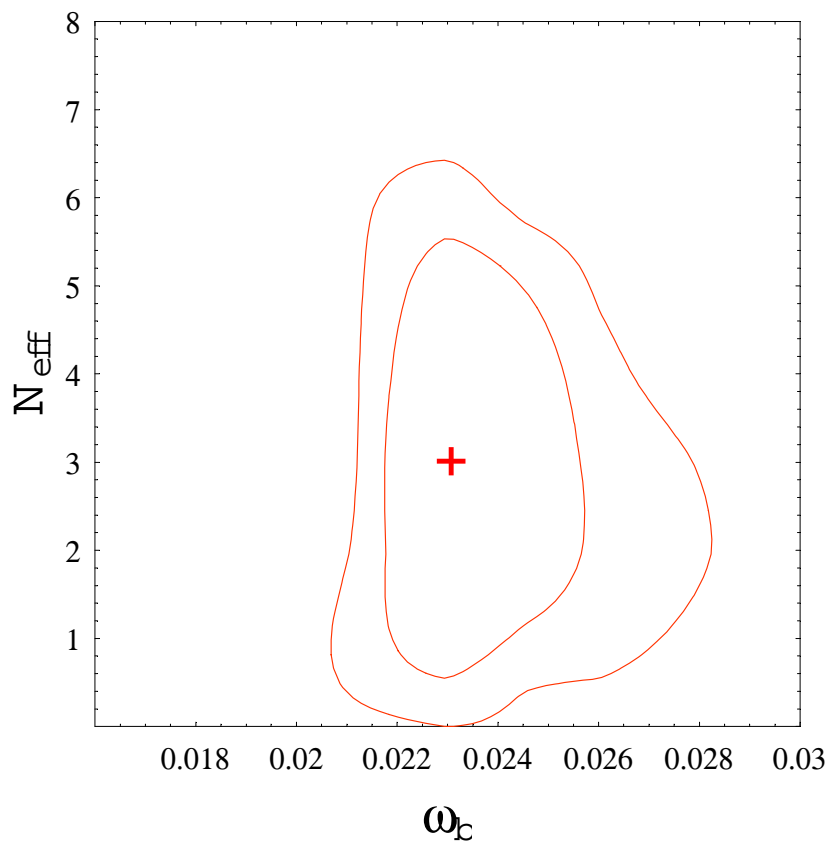

Figure 2: The 68 and 95\% C.L. likelihood contours from WMAP data in the $N_{e f f}-\omega_{b}$ plane.

temperature of the electromagnetic plasma is in the range $1 \div 0.01 \mathrm{MeV}$, and is strongly influenced by the two parameters $\Omega_{b} h^{2}$ and $N_{e f f}$. Increasing the value of the baryon to photon number density enhances the fusion mechanism, so it leads to a larger eventual amount 
of ${ }^{4} \mathrm{He}$, the most tightly bound light nucleus $\left({ }^{4} \mathrm{He}\right.$ binding energy per nucleon is of the order of $7 \mathrm{MeV}$ ). On the other hand, $D$ rapidly decreases with $\Omega_{b} h^{2}$, so the experimental result on this species is a very sensitive measure of baryons in the universe. The contribution of relativistic degrees of freedom to the expansion rate, parameterized by $N_{\text {eff }}$ affects instead the decoupling temperature of weak reaction which keep in chemical equilibrium protons and neutrons. For large temperatures in fact the ratio of their densities is given by equilibrium conditions, $n / p=\exp \left(-\left(m_{n}-m_{p}\right) / T\right)$, therefore if weak interactions were efficient down to very low temperatures, much smaller than the neutron-proton mass difference, neutrons would completely disappear. We mentioned however that the rate of these processes indeed becomes smaller than the expansion rate $H$ for temperatures of the order of $T_{D} \sim 1 \mathrm{MeV}$, so that the $n / p$ ratio freezes-out at the value $n / p=\exp \left(-\left(m_{n}-m_{p}\right) / T_{D}\right)$. Since almost all neutrons are eventually bound in ${ }^{4} \mathrm{He}$ nuclei it is then straightforward to get for the Helium mass fraction

$$
Y_{p} \equiv \frac{4 n_{H e}}{n_{b}} \sim 2 \frac{n / p}{1+n / p}=2 \frac{1}{e^{\left(m_{n}-m_{p}\right) / T_{D}}+1}
$$

When correcting this result for neutron spontaneous decay one gets $Y_{p} \sim 0.25$, already an excellent estimate compared with the result of detailed numerical calculations. Changing $N_{\text {eff }}$ affect the decoupling temperature $T_{D}$ and so the amount of primordial Helium.

An accurate analysis of BBN can be only achieved by numerically solving a set of coupled differential equations, taking into account quite a complicated network of nuclear reactions. Some of these reactions are still affected by large uncertainties, which therefore introduce an error in the theoretical prediction for, mainly, $D$ and ${ }^{7} L i$ abundances. As we mentioned Helium prediction is mainly influenced by $n \leftrightarrow p$ processes, which are presently known at a high level of accuracy $\left[\bar{G}_{\bar{Q}}\right]-\left[\overline{i_{i}}\right]$. Quite recently a big effort has been devoted in trying to quantify the role of each nuclear reaction to the uncertainties on nuclei abun-

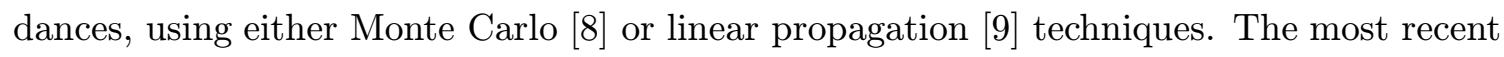

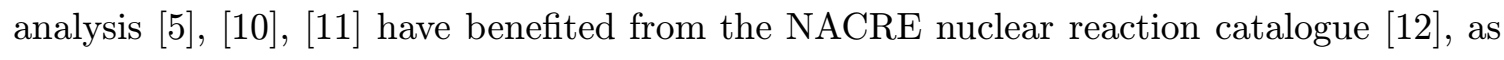
well as of very recent results, as for example the LUNA Collaboration measurement of the $D(p, \gamma)^{3} \mathrm{He}$ [i] uncertainties $\sigma_{i}^{\text {th }}$ on $Y_{p}$ and $D$ and ${ }^{7} L i$ number fractions $X_{i}=n_{i} / n_{b}$

$$
\frac{\sigma_{D}}{X_{D}} \sim 10 \%, \frac{\sigma_{H e}}{Y_{p}} \sim 0.1 \%, \frac{\sigma_{L i}}{X_{L i}} \sim 25 \%
$$

The large error on ${ }^{7} \mathrm{Li}$ is mainly due to the uncertainty on the rate for the ${ }^{4} \mathrm{He}\left({ }^{3} \mathrm{He}, \gamma\right){ }^{7} \mathrm{Be}$, a process which is also of great interest for the determination of both ${ }^{7} \mathrm{Be}$ and ${ }^{8} \mathrm{~B}$ neutrino fluxes from the sun. Hopefully it will be studied at low energies in the near future.

The experimental determination of primordial abundances is really a challenging task. The strategy is to identify metal poor environment, which are not been severely polluted by star contamination in their light nuclei content, and possibly to correct the observations for the effect of galactic evolution.

The ${ }^{4} \mathrm{He}$ mass fraction is determined by regression to zero metallicity of the values obtained by observing $\mathrm{HeII} \rightarrow \mathrm{HeI}$ recombination lines in extragalactic ionized gas. There 


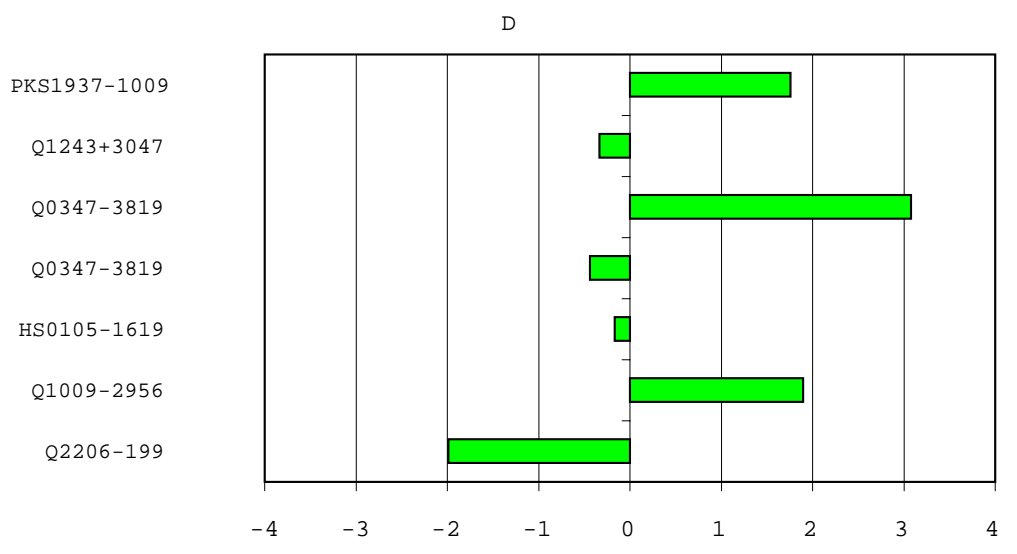

Figure 3: The pulls of QSO D measurements with respect to the theoretical prediction for $N_{\text {eff }}=$ 3.04 and $\omega_{b}=0.023$, in units of $\left(\left(\sigma_{D D}^{t h}\right)^{2}+\left(\sigma_{D}^{e x p}\right)^{2}\right)^{1 / 2}$.

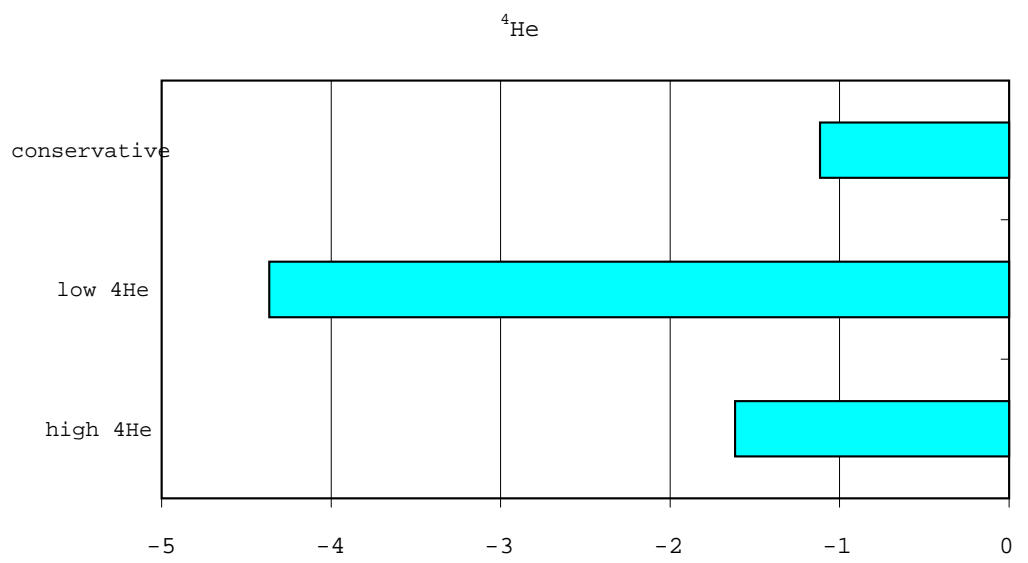

Figure 4: The pulls of $Y_{p}$ measurements with respect to the theoretical prediction for $N_{\text {eff }}=3.04$ and $\omega_{b}=0.023$, in units of $\left(\left(\sigma_{44}^{t h}\right)^{2}+\left(\sigma_{4}^{e x p}\right)^{2}\right)^{1 / 2}$.

are still quite different results (see e.g. [īin] for a review and references), a low one, $Y_{p}=$ $0.234 \pm 0.003$, and a high value, $Y_{p}=0.244 \pm 0.002$. In the following we also use a conservative estimate, $Y_{p}=0.239 \pm 0.008$.

The best estimate of Primordial $D$ comes from observations of absorption lines in gas clouds in the line of sight between the earth and Quasars at very high redshift $(z \sim$ $2-3)$, which give $X_{D}=\left(2.78_{-0.38}^{+0.44}\right) \cdot 10^{-5}\left[{ }_{1}^{1} \overline{5}_{1}\right]$. Finally ${ }^{7} \mathrm{Li}$ is measured via observation of absorption lines in spectra of POP II halo stars, which show a saturation of ${ }^{7} \mathrm{Li}$ abundance at low metallicity (Spite plateau).

The present status of BBN, in the standard scenario, using the value of baryon density as determined by WMAP and $N_{e f f}=3.04$ is summarized in Fig.s 3-5. Here I report the difference between the theoretical and the experimental determination, normalized to the total uncertainty, theoretical and experimental, summed in quadrature. The average of the several $D$ measurements, reported above, is indeed in very good agreement with theory. 
${ }^{7} \mathrm{Li}$

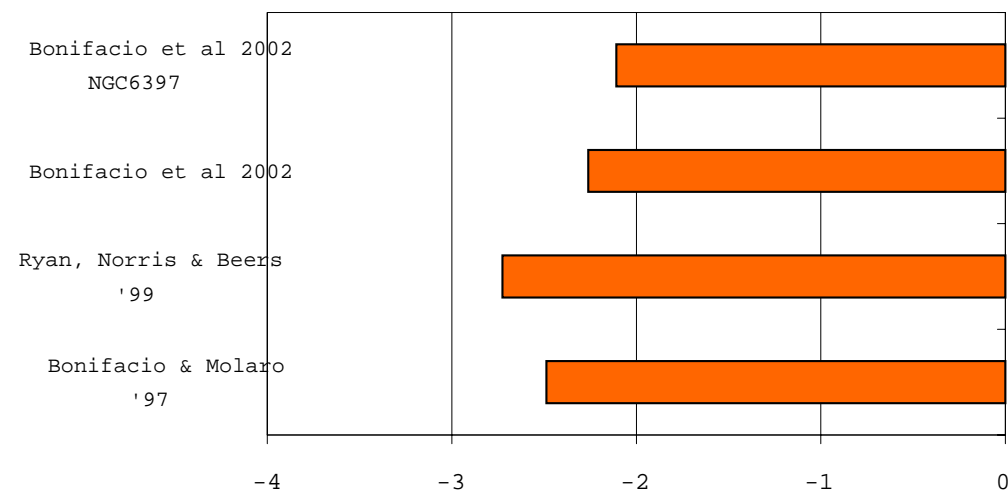

Figure 5: The pulls of $X_{7 L i}$ measurements with respect to the theoretical prediction for $N_{e f f}=$ 3.04 and $\omega_{b}=0.023$, in units of $\left(\left(\sigma_{77}^{t h}\right)^{2}+\left(\sigma_{7}^{e x p}\right)^{2}\right)^{1 / 2}$.

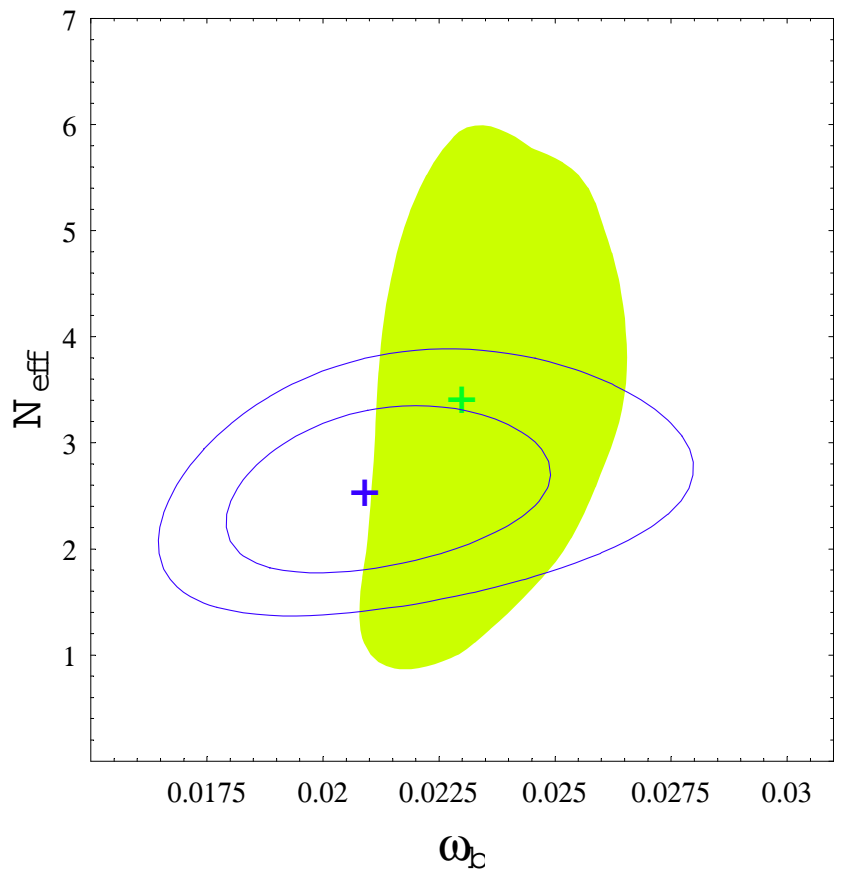

Figure 6: The 68 and $95 \%$ C.L. contours for the $D+{ }^{4} H e$ likelihood function in the $\omega_{b}-N_{\text {eff }}$ plane $\left(\omega_{b}=\Omega_{b} h^{2}\right)$. We also show the result of the $C M B+D$ analysis (colored area).

This is a very crucial result since, as we said already, $D$ is strongly influenced by $\Omega_{b} h^{2}$, which is now fixed by WMAP. In Fig. 6 I show the combined likelihood contours at $2 \sigma$ in the $N_{\text {eff }}-\Omega_{b} h^{2}$ plane obtained when using the WMAP result and $D$ measurement only (colored area) and the $\mathrm{D}+{ }^{4} \mathrm{He}$ results using the conservative $Y_{p}$ shown before.

It is evident that the effect of ${ }^{4} \mathrm{He}$ is to shift the values of both $\Omega_{b} h^{2}$ and $N_{\text {eff }}$ towards smaller values, which produces a smaller theoretical value for $Y_{p}$. Though this may be seen 
as a (weak) indication of the fact that a slightly lower value for $N_{\text {eff }}$ is preferred, I would more conservatively say that, waiting for a more clear understanding of possible systematics in $Y_{p}$ experimental determination, the standard scenario for $\mathrm{BBN}$ is in reasonable good shape. An open problem is however still represented by the evidence for ${ }^{7} \mathrm{Li}$ depletion, which is not fully understood (see Fig. 5). The theoretical result for $X_{L i}$ is in fact a factor 2-3 larger than the present experimental determination.

\section{Neutrino-antineutrino asymmetry}

While the electron-positron asymmetry density is severely constrained, of the order of $10^{-10}$ in unit of the photon density, we have no bounds at all on neutrino asymmetry from charge neutrality of the universe. Defining $\xi_{x}=\mu_{x} / T_{x}$, with $\mu_{x}$ the chemical potential for the $\nu_{x}$ species, with $x=e, \mu, \tau$, we recall that for a Fermi-Dirac distribution the particle-antiparticle asymmetry is simply related to $\xi_{x}$ (I assume here for simplicity massless neutrinos)

$$
n\left(\nu_{x}\right)-n\left(\bar{\nu}_{x}\right)=\frac{T_{x}^{3}}{6}\left(\xi_{x}+\frac{\xi_{x}^{3}}{\pi^{2}}\right)
$$

since neutrinos decoupled as hot relics starting from a chemical equilibrium condition with $e^{ \pm}$, so that $\mu_{x} \equiv \mu\left(\nu_{x}\right)=-\mu\left(\bar{\nu}_{x}\right)$. Non vanishing values for $\xi_{x}$ affects very weakly CMB, while it is much more constrained by BBN. In fact any asymmetry in the neutrino sector contribute to the Hubble expansion rate, i.e. to $N_{\text {eff }}$

$$
N_{e f f} \rightarrow N_{e f f}+\sum_{x}\left[\frac{30}{7}\left(\frac{\xi_{x}}{\pi}\right)^{2}+\frac{15}{7}\left(\frac{\xi_{x}}{\pi}\right)^{4}\right]
$$

In addition the asymmetry in the electron neutrino sector directly affects the $n / p$ value at the freeze-out of weak interactions, since they directly enter in the processes governing this phenomenon, namely $n+\nu_{e} \leftrightarrow p+e^{-}, n \leftrightarrow p+e^{-}+\bar{\nu}_{e}$ and $n+e^{+} \leftrightarrow p+\bar{\nu}_{e}$.

It was recently realized [i $\left[\begin{array}{l}1 \\ 6\end{array}\right]$ in that indeed, because of flavor oscillation, using present determination of mass differences and mixing angles from atmospheric and solar neutrinos, the three $\xi_{x}$ should be very close each other, so the bound on their (common) value $\xi$ mainly come from the fact that $\xi_{e}$ should be quite small $\xi \leq 0.1$, to give a value for the $n / p$ ratio (and so for ${ }^{4} \mathrm{He}$ ) in agreement with data. In Fig. 7 I show the likelihood contour obtained in the $\xi-N_{\text {eff }}$ plane [i.j]. Though the standard BBN is preferred, there is still room for very exotic scenarios, with larger neutrino degeneracies and even very large (or very small) $N_{\text {eff }}$.

\section{Cosmological bounds on neutrino mass scale}

Despite of the fact that we presently know neutrino mass differences from oscillation effects in atmospheric and solar neutrino fluxes, there is still quite a wide range for their absolute mass scale, spanning several order of magnitude, from few $\mathrm{eV}$ down to $10^{-2} \mathrm{eV}$. Terrestrial bounds come from Tritium decay experiments [i] $\bar{Z}_{-1} \overline{\bar{T}_{1}}$, which presently give $m\left(\nu_{e}\right) \leq 2.2 \mathrm{eV}$. 


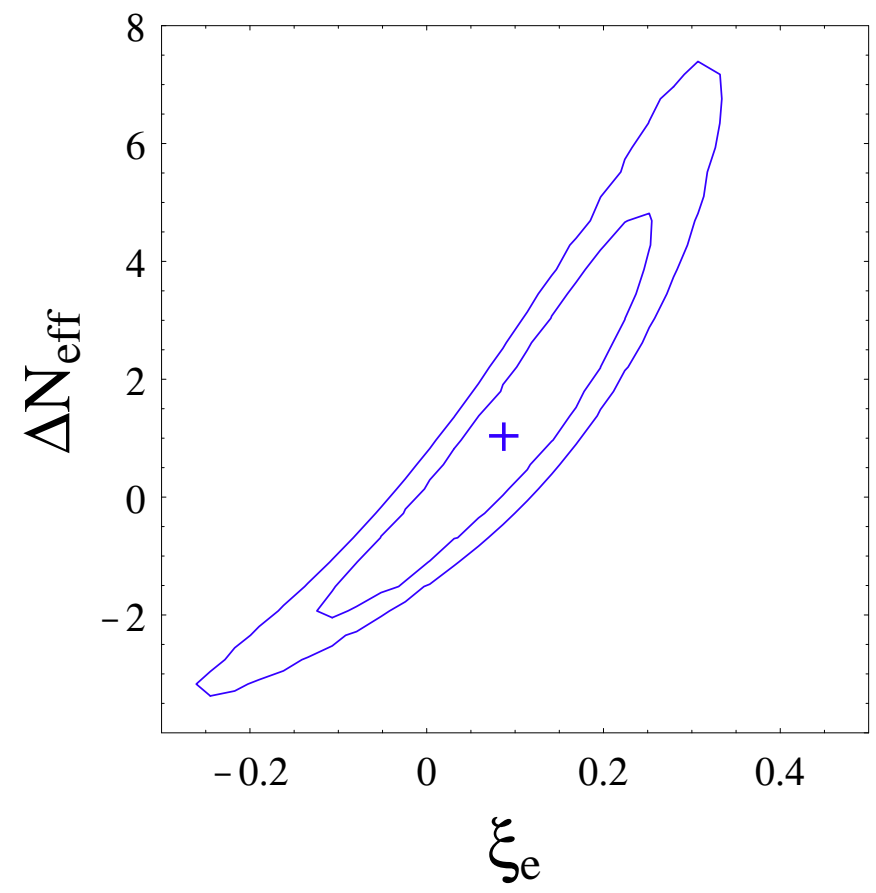

Figure 7: The 68 and $95 \%$ C.L. likelihood contours in the $\xi_{e}-\Delta N_{\text {eff }}$ plane from BBN, with $\Delta N_{\text {eff }}=N_{\text {eff }}-3.04$.

This result will be greatly improved by next generation experiment KATRIN, which should reach a sensitivity after three years of running of the order of $0.35 \mathrm{eV}$ [1]

An independent source of information will be provided by neutrinoless beta decay, which is sensitive to the effective $\nu_{e}$ mass

$$
<m_{e}>=\left|U_{e 1}\right|^{2} m_{1}+\left|U_{e 2}\right|^{2} e^{i \phi_{2}} m_{2}+\left|U_{e 3}\right|^{2} e^{i \phi_{3}} m_{3}
$$

with $U_{e i}$ the electron neutrino projection onto mass eigenstates with mass $m_{i}$, and $\phi_{i} \mathrm{CP}$

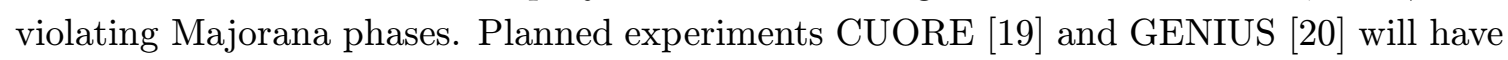
a sensitivity on this parameter of the order of $10^{-1}-10^{-2} \mathrm{eV}$.

Interestingly, quite severe constraints on neutrino masses come from cosmology. Massive neutrinos in fact contribute to the present total energy density of the Universe as $m_{\nu} n_{\nu}$ so we get

$$
\Omega_{\nu} h^{2}=\frac{\sum_{x} m\left(\nu_{x}\right)}{92.5 \mathrm{eV}}
$$

which gives a generous bound when imposing $\Omega_{\nu} h^{2}<1$.

Neutrino masses however also enter in the way structures grow for gravitational instability from the initial seed likely given by adiabatic perturbations produced during the inflationary phase. In fact neutrinos free stream and tend to suppress structure formation on all scales unless they are massive. In this case they can only affect scales smaller than the Hubble horizon when they eventually become non relativistic, the ones with a 
corresponding wave number larger than [2] $2 \overline{1}]$

$$
k_{n r} \sim 0.026\left(\frac{m_{\nu}}{1 e V}\right)^{1 / 2} \Omega_{m}^{1 / 2} h M p c^{-1}
$$

Several authors have recently considered this issue in details [202 2$]-[\overline{2} \overline{4}]$, combining WMAP data and the results of the 2dFGRS survey [25]. Actually the result also depends on the specific value of $N_{\text {eff }}$. A conservative value is given by $\sum_{x} m\left(\nu_{x}\right) \leq 2 \mathrm{eV}$.

\section{Acknowledgments}

It is a pleasure to thank the Committee of AHEP 2003 for invitation and support, and for the big effort in organizing such an interesting and stimulating conference.

\section{References}

[1] G. Mangano, G. Miele, S. Pastor, and M. Peloso, Phys. Lett. B534, 8 (2002).

[2] C.L. Bennett et al. (WMAP Coll.), Astrophys. J. Suppl. 148, 1 (2003).

[3] P. Crotty, J. Lesgourgues, and S. Pastor, Phys. Rev. D67, 123005 (2003).

[4] E. Pierpaoli, Mon. Not. Roy. Astron. Soc. 342, L63 (2003).

[5] A. Cuoco et al. ., astro-ph/0307213.

[6] R.E. Lopez and M.S. Turner, Phys. Rev. D59, 103502 (1999).

[7] S. Esposito, G. Mangano, G. Miele and O. Pisanti, Nucl. Phys. B568, 421 (2000).

[8] L.M. Krauss and P. Romanelli, Astrophys. J. 358, 47 (1990); P.J. Kernan and L.M. Krauss, Phys. Rev. Lett. 72, 3309 (1994).

[9] G. Fiorentini, E. Lisi, S. Sarkar, and F.L. Villante, Phys. Rev. D58, 063506 (1998).

[10] S. Burles, K.M. Nollett, and M.S. Turner, Astrophys. J. 552, L1 (2001).

[11] A. Coc, E. Vangioni-Flam, and M. Cassé, astro-ph/0002248; astro-ph/0009297; astro-ph/0101286.

[12] C. Angulo et al., Nucl. Phys. A656 (1999) 3. NACRE web site http://pntpm.ulb.ac.be/nacre.htm

[13] LUNA Collaboration, Nucl. Phys. A706 (2002) 203.

[14] B.D. Fields, and S. Sarkar, in Review of Particle Properties, Phys. Rev. D66, 010001 (2002).

[15] D. Kirkman, D. Tytler, N. Suzuki, J.M. O'Meara, and D. Lubin, astro-ph/0302006.

[16] A.D. Dolgov, S.H. Hansen, S. Pastor, S.T. Petcov, G.G. Raffelt, and D.V. Semikoz, Nucl. Phys. B362, 363 (2002).

[17] V.M. Lobashev et al. (Troisk Coll.), Phys. Lett. B460, 227 (1999); C. Weinheimer et al. (Mainz Coll.), Phys. Lett. B460, 219 (1999).

[18] A. Osipowicz et al., hep-ph/0109033.

[19] C. Arnaboldi et al. (CUORE Coll.), hep-ex/0212053. 
[20] H.V. Klapdor-Kleingrothaus (GENIUS Coll.), hep-ph/9910205.

[21] W. Hu, D.J. Eisenstein, and M. Tegmark, Phys. Rev. Lett. 80, 5255 (1998).

[22] D.N. Spergel et al., Astrophys. J. Suppl. 148, 175 (2003).

[23] O. Elgaroy and O. Lahav, JCAP 0304, 004 (2003).

[24] S. Hannestad, JCAP 0305, 004 (2003).

[25] J. Peacock et al., Nature 410, 169 (2001). 